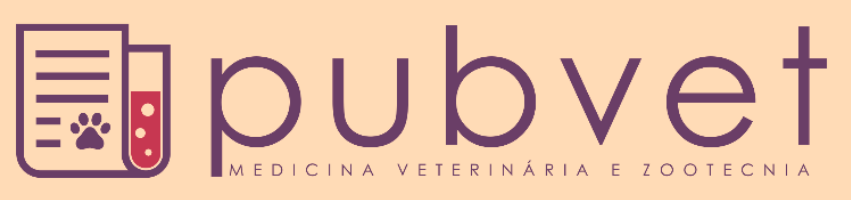

https://doi.org/10.31533/pubvet.v13n7a379.1-6

\title{
Avaliação do consumo e comportamento de bezerras da raça holandesa alimentadas com grão de milho (Zea mays L.) moído reidratado e ensilado ${ }^{1}$
}

Guilherme Martins de Almeida $\mathrm{Cruz}^{2}$, Renata Vitarele Gimenes Pereira ${ }^{3}$, Wellyngton Tadeu Vilela Carvalho $^{4 *}$, Queila Gouveia Tavares ${ }^{2}$, Lucas Vieira Silva ${ }^{2}{ }^{\circ}$, Janaína Aparecida Miranda Resende $^{20}$, Alvino Marcelo de Oliveira $^{20}$, Carlos Antunes Oliveira de Carvalho ${ }^{50}$, Gustavo Piacesi Rocha ${ }^{5}$, Aline Silva Oliveira ${ }^{5}$

${ }^{1}$ Trabalho de Conclusão de Curso do primeiro autor

${ }^{2}$ Eng. Agr., Instituto Federal do Sudeste de Minas Gerais - Campus Barbacena, Barbacena-MG, Barbacena-MG, Brasil.

${ }^{3}$ Méd. Vet., D. Sc., Professor do Instituto Federal do Sudeste de Minas Gerais - Campus Barbacena-MG, Brasil.

${ }^{4}$ Zootecnista, D. Sc., Professor do Instituto Federal do Sudeste de Minas Gerais - Campus Barbacena, - MG, Brasil.

${ }^{5}$ Graduando em Engenharia Agronômica pelo Instituto Federal do Sudeste de Minas Gerais - Campus Barbacena-MG, Brasil

*Autor para correspondência, E-mail: wellyngton.vilela@ifsudestemg.edu.br

Resumo. O milho é um dos cereais mais cultivados no mundo, sendo que a maior parte da produção é destinada a alimentação animal. Com o intuito de minimizar os problemas causados pelo armazenamento e visando obter uma maior eficiência na alimentação bovina com dietas a base de milho, vários métodos de processamento do grão vem sendo testados. O presente trabalho tem como objetivo avaliar o consumo e comportamento de bezerras da raça holandesa, alimentadas com grão de milho moído reidratado e ensilado, em substituição à ração formulada. O experimento foi conduzido no IF SUDESTE MG Campus Barbacena, sendo utilizadas quinze bezerras Holandesas em um delineamento inteiramente casualizado em parcelas subdivididas, sendo as parcelas, os três tratamentos (T1: 0\%, T2: $50 \%$ e T3: 100\% de substituição da ração formulada por silagem de grão de milho moído e reidratado), com cinco repetições, e as subparcelas os três períodos de $24 \mathrm{~h}$ para observação de comportamento. Os parâmetros de comportamento ócio, alimentando, dormindo, urinando, defecando e ingerindo água, como também o parâmetro referente ao consumo de alimento, não foram afetados $(\mathrm{P}>0,05)$ pelos tratamentos. Os parâmetros consumo de água, deitado e ruminando foram afetados $(\mathrm{P}<0,05)$, variando de 27,46 a 19,23 litros, 19,93 a $26,20 \%$ e 37,07 a $41,30 \%$, respectivamente. A substituição da ração formulada por silagem de grãos de milho reidratado e ensilado não provocou alterações no comportamento dos animais durante o período avaliado.

Palavras chave: conservação de concentrado, nutrição, ruminantes

\section{Evaluation of the intake and behavior of Holstein calves fed with ground corn (Zea mays L.) rehydrated and ensiled}

Abstract. Corn is one of the world's most widely grown cereals, with most of the production being fed. In order to minimize the problems caused by storage and to obtain a higher efficiency in the bovine feed with corn-based diets, several methods of grain processing have been tested. The objective of this study was to evaluate the consumption and behavior of Holstein heifers, fed with milled rehydrated and silage corn, instead of the formulated feed. The experiment was conducted at the IF SUDESTE MG - Campus Barbacena, and fifteen Holstein heifers were used in a completely randomized design in subdivided plots, the plots being the three treatments (T1: $0 \%, \mathrm{~T} 2=50 \%$ and $\mathrm{T} 3: 100 \%$ 
substitution of the feed formulated by milled and rehydrated corn grain silage), with five replications, and the subplots the three 24 hour periods for behavior observation. The idle behavior parameters, feeding, sleeping, urinating, and defecating only water but also the parameter relating to food consumption were not affected $(\mathrm{P}>0.05)$ by treatments. The water consumption parameters, and lying ruminating were affected $(\mathrm{P}<0.05)$, ranging from 27.46 to 19.23 liters 19.93 to $26.20 \%$ and from 37.07 to $41.30 \%$, respectively. Replacing the feed formulated by silage of rehydrated and ensiled corn kernels did not cause changes in the behavior of the animals during the evaluated period.

Key words: concentrate conservation, nutrition, ruminants

\title{
Evaluación de la ingesta y el comportamiento de terneros Holstein alimentados con maíz molido (Zea mays L.) rehidratado y ensilado
}

\begin{abstract}
Resumen. El maíz es uno de los cereales más cultivados en el mundo, y la mayor parte de la producción está destinada a la alimentación animal. Con el fin de minimizar los problemas causados por el almacenamiento y para lograr una mayor eficiencia en la alimentación bovina con dietas a base de maíz, varios métodos de procesamiento del grano vienen siendo probados. El presente trabajo tiene como objetivo evaluar el consumo y comportamiento de terneras de la raza holandesa, alimentadas con grano de maíz molido rehidratado y ensilado, en sustitución a la ración formulada. El experimento fue conducido en el IF SUDESTE MG - Campus Barbacena, siendo utilizadas quince terneras Holandesas en un delineamiento completamente casualizado en parcelas subdivididas, siendo las parcelas, los tres tratamientos (T1: 0\%, T2: 50\% y T3: 100\% de sustitución de la ración formulada por ensilaje de grano de maíz molido y rehidratado), con cinco repeticiones, y las subparcelas los tres períodos de $24 \mathrm{~h}$ para observación de comportamiento. Los parámetros de comportamiento ocios, alimentando, durmiendo, orinando, defecando e ingeriendo agua, como también el parámetro referente al consumo de alimento, no fueron afectados $(\mathrm{P}>0,05)$ por los tratamientos. Los parámetros de consumo de agua, acostado y rumiantes fueron afectados ( $\mathrm{P}<0,05$ ), variando de 27,46 a 19,23 litros, 19,93 a 26,20\% y 37,07 a 41,30\%, respectivamente. La sustitución de la ración formulada por ensilaje de granos de maíz rehidratado y ensilado no provocó cambios en el comportamiento de los animales durante el período evaluado.
\end{abstract}

Palabras clave: conservación de concentrado, nutrición, rumiantes

\section{Introdução}

O setor agropecuário apresenta grande importância social e econômica para o país, tendo grande participação sobre produto interno bruto, bem como sendo responsável pela geração de renda em grande parte das pequenas e médias propriedades rurais (ANUALPEC, 2018). No ano de 2015, o Brasil era responsável por $7 \%$ da produção mundial de leite com mais de 30 bilhões de litros de leite produzido (FAPRI, 2019) e em 2018 tal produção alcançou o patamar de 39 bilhões de litros (ANUALPEC, 2018). Devido ao aumento da produção leiteira do país, tem sido crescente a demanda por alimentos que possam atender as exigências nutricionais dos animais e/ou substituir um alimento que demande maior investimento de capital para sua produção. A busca por essas novas fontes de alimentação passam por pesquisa e seleção de forragens mais produtivas, utilização de forragens alternativas e de fontes já tradicionais na alimentação dos animais, como o milho (Zea mays L.) e grãos alternativos (Dawson, 2012; Prado et al., 2000). O milho constitui grande parte do alimento concentrado fornecido aos animais, sendo que, cerca de $70 \%$ da produção mundial deste é destinada à nutrição de animais (aves, bovinos, ovinos, entre outros) (Prado et al., 2010), permitindo assim, a adoção de novas formas de utilização, pode ser um fator primordial para maximizar a utilização desse alimento. Uma forma alternativa a utilização do milho moído é a sua ensilagem, onde o milho grão é reidratado e ensilado permitindo assim a melhora de suas características nutricionais.

Os estudos referentes ao comportamento animal são de grande importância para auxiliar o produtor na definição do manejo a ser adotado, proporcionando incrementos na produtividade (Dawkins, 2017; 
Llonch et al., 2015; Mendonça et al., 2004), principalmente em se tratando de novos alimentos e diferentes formas de conservação.

Dessa forma, objetiva-se com este trabalho avaliar o consumo e comportamento de bezerras da raça holandesa, alimentadas com grão de milho moído reidratado e ensilado, em substituição à ração formulada.

\section{Material e métodos}

O experimento foi conduzido no município de Barbacena - Minas Gerais (Latitude: $21^{\circ} 13^{\prime} 33^{\prime \prime} \mathrm{S}$, Longitude: $43^{\circ} 46^{\prime} 25^{\prime \prime} \mathrm{W}$ e altitude: 1164 metros), região da Serra da Mantiqueira, mesorregião do Campo das Vertentes, no Núcleo de Zootecnia do Instituto Federal de Educação, Ciência e Tecnologia do Sudeste de Minas Gerais - Campus Barbacena. Segundo a classificação climática de Köppen \& Geiger (1928), a região apresenta um clima tropical de altitude (Cwb), sendo que, a média de temperatura no período de primavera/verão varia de $16,9^{\circ}$ a $19,9^{\circ} \mathrm{C}$, e de 14,4 a $17,5^{\circ} \mathrm{C}$ no outono/inverno. O índice pluviométrico no período da primavera/verão é de $1143,6 \mathrm{~mm}$ e de $206,5 \mathrm{~mm}$ no outono/inverno (EMBRAPA, 2006). O experimento foi realizado no mês de setembro de $2018 \mathrm{com}$ temperaturas médias de $20,6 \pm 0,7{ }^{\circ} \mathrm{C}$ e umidade relativa do ar de $90 \%$.

Para realização do experimento foram utilizadas 15 bezerras Holandesas com peso médio de 255,26 $\pm 44,44 \mathrm{~kg}$ de peso vivo. Sendo elas divididas em três tratamentos em função do nível substituição da ração formulada pela silagem de grão de milho reidratado $(0,50$ e 100\%). Ao início do experimento todos os animais foram tratados contra endo e ectoparasitas (vermífugo via oral e carrapaticida Pour on). Os animais foram alojados em camas individuais, em sistema do tipo tie stall dotadas de comedouros e bebedouros individuais. As dietas foram fornecidas duas vezes ao dia (09:00 e 16:00 horas).

A silagem de grão de milho reidratado foi produzida no próprio Instituto, sendo os grãos de milho moídos, reidratados á $44 \%$ de umidade e depois acondicionados em um recipiente plástico (caixa d'água com capacidade para 1.000 litros), fechado e vedado de forma a gerar um ambiente anaeróbico em seu interior, por um período de quinze dias. Permitindo assim, a fermentação dos grãos de milho.

As dietas fornecidas aos animais foram calculadas de acordo com os valores nutricionais dos alimentos fornecidos de modo a suprir a mantença dos animais e proporcionar ganho de peso aos mesmos (Tabela 1).

Tabela 1. Dieta em (g) da matéria natural fornecida diariamente às bezerras holandesas em relação ao peso médio inicial

\begin{tabular}{lccc}
\hline Alimento & Tratamento 1 $(0 \%)$ & Tratamento 2 (50\%) & Tratamento 3 (100\%) \\
\hline Silagem de milho & 12000 & 12000 & 12000 \\
Ração & 2000 & 1000 & - \\
SGMH & - & 2000 & 4000 \\
\hline
\end{tabular}

SGMH: silagem de grão de milho reidratado; (0\%), (50\%), (100\%): porcentagem de inclusão de SGMH

$\mathrm{O}$ experimento teve duração de 33 dias, sendo 15 dias de ensilagem do grão de milho moído e reidratado, 15 dias de adaptação dos animais as dietas e os três últimos dias destinados à avaliação do comportamento e consumo animal. Sendo o consumo de alimento, determinado através da diferença entre o fornecido e as sobras no cocho, nas 24 horas posteriores ao fornecimento. O consumo de água foi determinado quatro vezes ao dia, através da quantidade necessária para completar os bebedouros com capacidade para doze litros.

Na coleta de dados referentes ao comportamento, os animais foram observados simultaneamente, por três dias consecutivos, registrando-se a atividade ao final de cada intervalo de dez minutos (Mendonça et al., 2004), por meio de observação direta. Todas as atividades foram avaliadas em função do tempo. Foram mensurados os parâmetros tempo de ócio, deitado, alimentando, bebendo água, ruminando, defecando, urinando, dormindo e outros (Johnson \& Combs, 1991). Sendo caracterizado como "outros" o tempo em que as bezerras passaram realizando atividades que não se encaixam nos demais parâmetros.

Foi utilizado um delineamento inteiramente casualizado em parcelas subdivididas sendo as parcelas, os três tratamentos (T1: 0\%, T2: $50 \%$ e T3: 100\% de substituição da ração formulada por silagem de grão de milho reidratado), com cinco repetições e as sub parcelas, os três períodos de $24 \mathrm{~h}$, para observação de comportamento. 
Os dados referentes às médias dos tempos de alimentação, ruminação, tempo de ócio, deitado e outros, como também os dados referentes ao consumo, foram submetidos à análise de variância e as médias foram comparadas pelo teste SNK através do Sistema de Análises de Variância para Dados Balanceados - SISVAR ${ }^{\circledR}$ (Ferreira, 2011).

Para as frequências de ingestão de água, defecar e urinar foram realizadas as médias do número de ocorrência dos três dias de observação, pois alguns parâmetros apresentaram ocorrência zero, devido ao registro da atividade ser realizado quando ocorria ao final do intervalo amostral. Para todos estes parâmetros utilizou-se a análise não paramétrica e teste de comparação múltipla de Dunn para comparação das médias pelo programa GrafPad InStat ${ }^{\mathrm{TM}}$ (versão 3.10 - 2009).

\section{Resultados e discussão}

O consumo de água e de matéria seca por dia de avaliação está apresentado na tabela 2 . O consumo de água pelos animais foi influenciado pelas dietas, variando entre 27,46 e 19,23 L em 24 horas. Isto pode estar relacionado com o maior teor de umidade da silagem de grão. Sendo que Huffman (1959) em experimento com animais de diferentes composições genéticas, afirmam que o consumo de água esta relacionado positivamente com o consumo de matéria seca.

Tabela 2. Consumo de água e consumo de matéria seca $\left(\mathrm{kg} \mathrm{MS}^{-1}\right)$ das bezerras quando alimentadas com $100 \%$ de ração formulada (I) ou com silagem de grão de milho reidratado nas proporções de $50 \%$ (II) e $100 \%$ (III)

\begin{tabular}{lccc}
\hline & & Consumo de água (L) & CMS (kg) \\
\hline Silagem de grão de milho reidratado 00\% & I & $27,46 \mathrm{~A}$ & 6,06 \\
Silagem de grão de milho reidratado 50\% & II & $19,23 \mathrm{~B}$ & 5,85 \\
Silagem de grão de milho reidratado 100\% & III & $23,33 \mathrm{~A}$ & 5,97 \\
\hline
\end{tabular}

CMS: Consumo de matéria seca $(\mathrm{kg})$.

As dietas fornecidas não influenciaram $(\mathrm{P}>0,05)$ os tempos de alimentação dos animais (Tabela 3) em concordância com os resultados encontrados por (Carvalho et al., 2012) trabalhando com bezerras alimentadas com silagem pré-secada de Tifton 85 (Cynodon spp.) e também com os resultados de Lima (2016) os quais avaliaram o fornecimento de feno de botão de ouro (Tithonia diversifolia) para bezerras. Ressaltando que Carvalho et al. (2012) e Lima (2016) trabalharam com animais de estrutura corporal semelhante ao presente estudo e na mesma região. Bürger et al. (2000), avaliando o comportamento de bezerras alimentadas com diferentes níveis de inclusão de concentrados na dieta observou que os animais passavam entre 8,0 e $20,5 \%$ do dia alimentando-se. Sendo que os valores encontrados no presente estudo enquadram-se dentro deste perfil, devido à variação no nível de inclusão de concentrado nas dietas avaliadas, sendo observadas médias de 10,27 a 12,82\% do dia destinado à alimentação.

Tabela 3. Médias do tempo em porcentagem diária (\%), em que as bezerras permaneceram alimentando, ruminando, dormindo, em ócio, e realizando outras atividades, quando alimentadas com $100 \%$ de ração formulada (I) ou com silagem de grão de milho reidratado nas proporções de 50\% (II), e 100\% (III)

\begin{tabular}{lcccccc}
\hline \multirow{2}{*}{ Tratamentos } & \multicolumn{5}{c}{ Comportamento, \% } \\
\cline { 2 - 7 } & Alimentando & Ócio & Dormindo & Deitado & Ruminando & Outros \\
\hline I & 10,27 & 15,37 & 3,56 & $26,20^{\mathrm{A}}$ & $34,07^{\mathrm{B}}$ & 6,67 \\
II & 10,92 & 13,65 & 4,26 & $21,01^{\mathrm{AB}}$ & $39,86^{\mathrm{A}}$ & 6,94 \\
III & 12,82 & 12,36 & 4,63 & $19,93^{\mathrm{B}}$ & $41,30^{\mathrm{A}}$ & 8,19 \\
$P$-valores & & & & & & \\
& $\mathrm{ns}$ & $\mathrm{ns}$ & $\mathrm{ns}$ & 0,0408 & 0,0171 & $\mathrm{~ns}$ \\
\hline
\end{tabular}

ns: não significativo $(\mathrm{P}>0,05)$

O tempo em que os animais passaram dormindo, ócio e outros (brincar, observar, entre outros) não foi influenciado pelas dietas ( $\mathrm{P}>0,05)$. Miotto et al. (2014) descreveram que o tempo que tourinhos alimentados com diferentes níveis de inclusão de gérmen de milho, passavam dormindo foi de 7,75\% em 24 horas. Os valores obtidos no presente estudo foram inferiores aos observados por Miotto et al. (2014). Isto pode estar relacionado à diferença entre os grupos de animais avaliados. 
Pinto et al. (2010) avaliando o comportamento de tourinhos em função de três dietas, observaram períodos de ócio entre 53,9 e 55,1\% em 24 horas. Pinheiro et al. (2011) avaliando a inclusão de resíduos de mandioca também observaram elevados períodos de ócio (41,2\% em 24 horas). Os valores encontrados pelos autores citados anteriormente são muitos superiores ao encontrados no presente estudo, uma vez que os autores consideraram como ócio o tempo despendido pelos animais dormindo, deitados e em outras atividades.

O período em que os animais passaram deitados foi influenciado pelas dietas $(\mathrm{P}=0,0408)$, variando entre 19,93 e 26,20\% em 24 horas. Sendo estes valores superiores aos observados por Pinto et al. (2010) que foram de 4,62 a 4,78\%, em 24 horas, que utilizou também animais em sistema de confinamento. Isto pode estar relacionado com o manejo diário das baias, uma vez que as camas foram substituídas sempre que necessário.

O período despendido pelos animais para ruminação foi influenciado pelas dietas $(\mathrm{P}=0,0171)$. Sendo observado o aumento no período de ruminação com a adição da silagem de grão de milho reidratado. Isto pode estar relacionado com o aumento de energia fornecida pela silagem de grão, sendo uma fonte de energia mais disponível para os microrganismos iniciarem a degradação das frações fibrosas oriundas principalmente do volumoso. minutos Mendonça et al. (2004) observou tempo médio de ruminação de $35,04 \%$ em 24 horas para silagem de milho e 32,83 para cana-de-açúcar com concentrado (relação volumoso / concentrado: 60/40).

A frequência com que os animais ingeriram água, defecaram e urinaram não sofreu interferência das dietas $(\mathrm{P}>0,05)$ em relação ao consumo de ração (Tabela 4), isto pode sugerir que a silagem de grãos de milho moído e reidratado não provocou alterações digestivas e urinárias durante o período experimental.

Tabela 4: Médias e medianas da frequência (número de ocorrência em 24 horas) em que as bezerras beberam água, defecaram e urinaram quando alimentadas com $100 \%$ de ração formulada (I) ou com silagem de grão de milho reidratado nas proporções de $50 \%$ (II), e $100 \%$ (III).

\begin{tabular}{lcccc}
\hline & & \multicolumn{3}{c}{ Comportamento } \\
\cline { 3 - 5 } & & Água & Defecando & Urinando \\
\hline \multirow{2}{*}{ Médias } & I & 1,64 & 0,37 & 0,79 \\
& II & 1,71 & 0,32 & 0,83 \\
& III & 1,73 & 0,55 & 0,83 \\
\hline \multirow{2}{*}{ Medianas } & I & $1,39(0,00-4,17)$ & $0,00(0,00-1,39)$ & $0,69(0,00-2,08)$ \\
\multirow{2}{*}{ Significância } & II & $1,39(0,00-5,56)$ & $0,00(0,00-1,39)$ & $0,69(0,00-2,08)$ \\
\hline
\end{tabular}

ns: não significativo $(\mathrm{P}>0,05)$

\section{Referências bibliográficas}

ANUALPEC. (2018). Anuário da Pecuária Brasileira (20th ed. Vol. 1). São Paulo, São Paulo, Brasil: Instituto FNP.

Bürger, P. J., Pereira, J. C., Queiroz, A. C., Coelho, J. F., Agostini, P. S., Valadares Filho, S. C. \& Casali, A. D. P. (2000). Comportamento ingestivo em bezerros holandeses alimentados com dietas contendo diferentes níveis de concentrado. Revista Brasileira de Zootecnia, 29(1):236-242. doi: http://dx.doi.org/10.1590/S1516-35982000000100031.

Carvalho, W. T. V., Villanova, D. F. Q., Minighin, D. C., Lima, W. B. G., Matos Teixeira, A. \& Pereira, R. V. G. (2012). Comportamento de bezerras da raça Holandesa alimentadas com silagem pré-secada do capim Tifton 85 (Cynodon ssp.). PUBVET, 12(2):1-7.

Dawkins, M. S. (2017). Animal welfare and efficient farming: is conflict inevitable? Animal Production Science, 57(2):201-208. 
Dawson, L. E. R. (2012). The effect of inclusion of lupins/triticale whole crop silage in the diet of winter finishing beef cattle on their performance and meat quality at two levels of concentrates. Animal Feed Science and Technology, 171(2-4):75-84. doi: http://dx.doi.org/10.1016/j.anifeedsci.2011.09.011

EMBRAPA. (2006). Sistema brasileiro de classificação de solos (Vol. 412).

FAPRI. (2019). Food and Agricultural Policy Research Institute. Food and Agricultural Policy Research Institute, from http://www.fapri.iastate.edu/tools/outlook.aspx

Ferreira, D. F. (2011). SISVAR: A Computer Statistical Analysis System. Ciência e Agrotecnologia, 35(6):1039-1042. doi: http://dx.doi.org/10.1590/S1413-70542011000600001.

Huffman, C. F. (1959). Summer Feeding of Dairy Cattle. A Review. Journal of Dairy Science, 42(9):1495-1551. doi: http://dx.doi.org/10.3168/jds.S0022-0302(59)90766-0

Johnson, T. R. \& Combs, D. K. (1991). Effects of prepartum diet, inert rumen bulk, and dietary polyethylene glycol on dry matter intake of lactating dairy cows. Journal of Dairy Science, 74(3):933-944. doi: http://dx.doi.org/10.3168/jds.S0022-0302(91)78243-X

Köppen, W. \& Geiger, R. (1928). Klimate der Erde. Gotha: Verlag Justus Perthes. Wall-map $150 \mathrm{~cm} \times 200 \mathrm{~cm}$.

Lima, W. B. G. (2016). Consumo, comportamento e parâmetros clínicos de bezerras da raça holandesa alimentadas com feno de botão de ouro (Tithonia diversifolia). Barbacena, Minas Gerais, Brasil: Instituto Federal do Sudeste de Minas Gerais.

Llonch, P., King, E. M., Clarke, K. A., Downes, J. M. \& Green, L. E. (2015). A systematic review of animal based indicators of sheep welfare on farm, at market and during transport, and qualitative appraisal of their validity and feasibility for use in UK abattoirs. The Veterinary Journal, 206(3):289-297.

Mendonça, S. S., Campos, J. M. S., Valadares Filho, S. C., Valadares, R. F. D., Soares, C. A., Lana, R. P. \& Pereira, M. L. A. (2004). Comportamento ingestivo de vacas leiteiras alimentadas com dietas à base de cana-de-açúcar ou silagem de milho. Revista Brasileira de Zootecnia, 33(3):723-728.

Miotto, F. R. C., Neiva, J. N. M., Restle, J., Silva Falcão, A. J., Castro, K. J. \& Maciel, R. P. (2014). Comportamento ingestivo de tourinhos alimentados com dietas contendo níveis de gérmen de milho integral. Ciência Animal Brasileira, 15(1):45-54.

Pinheiro, A. A., Veloso, C. M., Santana Júnior, H. A., Rocha Neto, A. L., Silva, R. R., Lima, F. B. M. \& Cecato, U. (2011). Avaliação dos intervalos e números de observações no comportamento ingestivo de novilhas leiteiras confinadas. Revista Brasileira de Saúde e Produção Animal, 12(2):480-490.

Pinto, A. P., Marques, J. A., Abrahão, J. J. S., Nascimento, W. G., Costa, M. A. T. \& Lugão, S. M. B. (2010). Comportamento e eficiência ingestiva de tourinhos mestiços confinados com três dietas diferentes. Archivos de Zootecnia, 59(227):427-434.

Prado, I. N., Moreira, F. B., Prado, R. M. \& Rotta, P. P. (2010). Alimentos usados para supelementação de bovinos de corte. In I. N. Prado (Ed.), Produção de bovinos de corte e qualidade da carne (Vol. 1, pp. 73-148). Maringá, Paraná, Brasil: Eduem.

Prado, I. N., Nascimento, W. G., Zeoula, L. M., Alcalde, C. R., Medroni, S. \& Vinocur, K. (2000). Níveis de triticale em substituição ao milho no desempenho zootécnico e digestibilidade aparente de novilhas Nelore confinadas. Revista Brasileira de Zootecnia, 29(5):1545-1552.

Recebido: 30 de maio, 2019.

Aprovado: 1 de julho, 2019.

Publicado: 12 de agosto, 2019.

Licenciamento: Este artigo é publicado na modalidade Acesso Aberto sob a licença Creative Commons Atribuição 4.0 (CC-BY 4.0), a qual permite uso irrestrito, distribuição, reprodução em qualquer meio, desde que o autor e a fonte sejam devidamente creditados. 